\title{
O PROTAGONISMO DA ENFERMAGEM NO COMBATE DO COVID-19
}

\section{ARTIGO DE REVISÃO}

CESÁRIO, Jonas Magno dos Santos ${ }^{1}$

FLAUZINO, Victor Hugo de Paula ${ }^{2}$

MEJIA, Judith Victoria Castillo ${ }^{3}$

LOPES, Kedma Carolina Ferreira ${ }^{4}$

CESÁRIO, Jonas Magno dos Santos. Et al. O protagonismo da enfermagem no combate do COVID-19. Revista Científica Multidisciplinar Núcleo do Conhecimento. Ano 05, Ed. 11, Vol. 25, pp. 149-168. Novembro de 2020. ISSN: 2448-0959, Link de acesso: https://www.nucleodoconhecimento.com.br/saude/protagonismo-daenfermagem

1 Mestrado em Medicina. Especialização em andamento em Engenharia e gerenciamento de manutenção. Especialização em andamento em Engenharia eletrônica e de computação. Especialização em Auditoria em Serviço de Enfermagem. Especialização em Docência para o Ensino Profissionalizante. Especialização em Formação de docentes para o ensino em Enfermagem. Especialização em Enfermagem em Emergência e Urgência. Graduação em andamento em Engenharia de Software. Graduação em Enfermagem.

2 Enfermeiro, possui graduação em Enfermagem pela Universidade de Mogi das Cruzes. Especialista em Centro Cirúrgico e Central de Material pela Universidade Nove de Julho. Pós-graduando dos cursos de Docência para Enfermagem e Enfermagem em Neurocirurgia.

${ }^{3}$ Mestrado em Enfermagem. Graduação em enfermagem.

${ }^{4}$ Graduação em Enfermagem. 


\section{RESUMO}

Objetivou-se neste estudo descrever o protagonismo da enfermagem no combate do covid-19. Tratou-se de uma revisão integrativa da literatura, de abordagem descritiva e qualitativa, elaborada no mês de setembro/2020 com os seguintes descritores: SARS-Cov-2, COVID-19 e Atuação da Enfermagem. Buscou-se reunir evidências para responder a seguinte pergunta de pesquisa: Qual foi o protagonismo da enfermagem no combate da COVID-19? Incluiu-se artigos acadêmicos publicados no 2019 e 2020 na língua portuguesa, disponíveis de forma gratuita nos bancos de dados da SCIELO (Scientific Electronic Library Online), LILACS (Literatura Latino-Americana e do Caribe em Ciências da Saúde) e BVS (Biblioteca Virtual de Saúde), resultando numa amostra final de 51 artigos. Os resultados dos estudos mostram que o principal protagonismo foi o processo de humanização, além de ter o papel fundamental na criação de diversas campanhas educativas e criação de aplicativos, destaca-se ainda que a enfermagem se apresenta como força expressiva e completamente necessária no enfrentamento do novo COVID-19.

Palavras-chave: SARS-Cov-2, COVID-19, atuação da enfermagem.

\section{INTRODUÇÃO}

Algumas pandemias podem desencadear graves problemas de saúde pública e levar os países afetados a crises políticas e socioeconômicas desastrosas. O novo Coronavírus se tornou uma grande ameaça para a saúde pública mundial. O surto deste vírus começou no mercado de frutos do mar de Huanan, situado na cidade Wuhan na China em dezembro de 2019. Rapidamente em alguns meses virou uma emergência de saúde mundial, afetando todos os continentes exceto a Antártica. Sendo categorizada pela a organização mundial de saúde (OMS) como uma pandemia mundial (AMITRANO; MAGALHÃES; SILVA, 2019; MINISTÉRIO DA SAÚDE, 2020).

Buscando combater esse surto, os países começaram a implantar medidas conforme orientações da OMS, como ser: isolamento social, fechamento do comercio não 
essencial e suspensão das aulas. Mas enquanto os líderes de diversos países preocupavam-se em salvar vidas, o presidente brasileiro Jair Messias Bolsonaro ia na contramão de todas as orientações da OMS, priorizando a economia e ridicularizando a COVID-19 (BORGES et al, 2020; OLIVEIRA; LUCAS; IQUIAPAZA, 2020).

Nesse cenário, a população no geral ficou dividida sobre qual decisão seguir, se a ciência ou a ideologia do presidente, dando origem a uma crise política e social. Os governadores estaduais e municipais tiveram que iniciar suas próprias medidas de combate ao Coronavirus, sem o apoio do Presidente da República (BORGES et al, 2020; FRANZOI; CAUDURO, 2020; OLIVEIRA; LUCAS; IQUIAPAZA, 2020).

Os profissionais de enfermagem não ficaram fora dessa situação crítica, pois foram afetados diretamente no aumento dos números de casos, gerando para a categoria da enfermagem problemas como: jornada de trabalho exaustiva, falta de equipamento de proteção individual (EPI) e leitos, profissionais de enfermagem escassos, déficit na verba e treinamento para lidar com o novo Coronavírus (COSTA, 2020; DAL'BOSCOI et al, 2020; NETO; BORTOLUZZI; FREITAS, 2020; SOUZA; SOUZA, 2020).

O ano de 2020 foi dedicado para enfermagem, pois nele é celebrado o $200^{\circ}$ aniversário do nascimento de Florence Nightingale. Assim sendo, a sociedade passou enxergar a essencialidade da enfermagem, aumentando sua valorização perante ela. Reconhece-se que a enfermagem está superando diversos desafios diariamente, dentre eles, ter que deixar seus lares para combater um novo vírus ainda desconhecido, enfrentando o medo de se tornar um número nas estatísticas de casos infectados e mortes, passando horas exaustivas de plantão, com suas faces marcadas pelo uso intenso de máscaras e muitos inclusive, tendo que dormir em hotéis, para evitar o contato com seus familiares devido ao risco de contaminação (COSTA et al, 2020; SILVA, 2020; SILVA et al, 2020(b)).

Um dado triste é que mais de 30 mil profissionais de enfermagem foram contaminados e 226 vieram a óbito pela COVID-19. O mundo também foi cenário de diversas homenagens, aplausos nas ruas e palavras de agradecimento pela dedicação e os cuidados prestados desses profissionais, contudo, a sociedade seja ela como 
paciente ou expectador pode perceber e reconhecer, como a Enfermagem é fundamental (BARRETO et al, 2020; GALLASCHI et al, 2020; MIRANDA et al, 2020; RIBOLI; ARTHUR; MANTOVANI, 2020).

Hoje em dia, sabe-se que a enfermagem está em evidência, pois além de ser o ano da enfermagem, se tem uma grande guerra contra um inimigo invisível, no qual os profissionais estão colocando a prova a sua saúde física e mental, mostrando as dificuldades diárias de lidar com uma doença nova, pouco conhecida e que está ceifando diversas vidas diariamente (GALLASCHI et al, 2020; FRANZOI; CAUDURO, 2020; HUMEREZ; OHL; SILVA, 2020; MEDEIROS, 2020)

Nesse contexto e perante essas homenagens surgiu a dúvida, qual foi o verdadeiro protagonismo da enfermagem durante o combate do novo Coronavírus? Contudo, o objetivo deste estudo foi o descrever o protagonismo da enfermagem no combate do Covid-19.

\section{METODOLOGIA}

Tratou-se de uma revisão integrativa da literatura, de abordagem descritiva e qualitativa. Segundo Cesário, Flauzino e Mejia (2020), a revisão integrativa da literatura consiste no procedimento sistemático e racional que tem como princípio resolver os problemas estabelecidos e com etapas preestabelecidas.

Na primeira etapa, buscou-se reunir evidências para responder a seguinte pergunta de pesquisa: Qual foi o protagonismo da enfermagem no combate da COVID-19 encontrado nas produções cientificas publicadas em 2019 e $2020 ?$

Na seguinte etapa definiu-se os seguintes descritores encontrados nos Descritores en Ciências da Saúde (DeCS), utilizando-os junto ao operador Booleano "AND", resultando da seguinte forma: SARS-Cov-2 AND COVID-19 AND Atuação da Enfermagem. Os bancos de dados utilizados foram SCIELO (Scientific Electronic Library Online), LILACS (Literatura Latino-Americana e do Caribe em Ciências da Saúde) e BVS (Biblioteca Virtual de Saúde). 
$\mathrm{Na}$ terceira etapa, estabeleceu-se como critérios de inclusão, os artigos acadêmicos publicados no 2019 e 2020, na língua portuguesa, disponíveis de forma gratuita e nos bancos de dados já mencionados, que respondessem à pergunta de pesquisa. Excluíram-se artigos duplicados e incompletos. A coleta dos dados foi realizada no mês de setembro/2020, por quatro pesquisadores de forma independente.

Encontraram-se no total 867 artigos, porém, após aplicação dos filtros mencionados, leitura dos resumos e a exclusão dos trabalhos duplicados/incompletos, resultou em 62 artigos, os quais foram lidos na integra. Destes 62 artigos, foram excluídos os que não respondiam à questão norteadora de pesquisa, resultando numa amostra final de 51 artigos, sendo apresentados em forma de tabela.

\section{RESULTADOS}

Por meio da tabela 1 é possível constatar a distribuição inicial dos artigos científicos encontrados nas bases de dados da LILACS, SCIELO e BVS.

Tabela 1 - Resultados da busca nas bases de dados

\begin{tabular}{|l|l|l|}
\hline Base de dados & \multicolumn{2}{|l|}{ Artigos } \\
\cline { 2 - 3 } & Total & Incluídos \\
\hline LILACS & 569 & 21 \\
\hline SCIELO & 59 & 21 \\
\hline BVS & 239 & 20 \\
\hline Total & $\mathbf{8 6 7}$ & $\mathbf{6 2}$ \\
\hline
\end{tabular}

Fonte: Elaborado pelos autores (2020).

Verifica-se que ano 2020 contém a maioria das publicações. Com relação ao tipo de artigo, constata-se que destes, $77 \%$ dos artigos foram originais e $33 \%$ foram de revisão da literatura. Quanto ao método, 55\% foram apresentaram abordagem quantitativa, $44 \%$ qualitativa e $11 \%$ foram do tipo misto (quantitativos e qualitativos). 
Para um melhor entendimento das publicações, foi elaborado o quadro 1, apresentando uma síntese dos artigos selecionados e contendo as variáveis número do artigo, autor, título e objetivo central.

Quadro 1 - Artigos inclusos neste estudo

\begin{tabular}{|c|c|c|c|}
\hline Vo & utor & Titulo do trabalho & o central \\
\hline 1. & $\begin{array}{l}\text { AMITRANO; } \\
\text { MAGALHÃES; } \\
\text { SILVA, } 2019\end{array}$ & $\begin{array}{l}\text { Medidas de } \\
\text { enfrentamento dos } \\
\text { efeitos econômicos da } \\
\text { pandemia COVID-19: } \\
\text { panorama } \\
\text { internacional e análise } \\
\text { dos casos dos } \\
\text { Estados Unidos, do } \\
\text { Reino unido e da } \\
\text { Espanha }\end{array}$ & $\begin{array}{l}\text { Apresentar um panorama } \\
\text { internacional das medidas } \\
\text { econômicas adotadas para } \\
\text { reduzir os graves efeitos } \\
\text { econômicos da pandemia de } \\
\text { Sars-COV-2. }\end{array}$ \\
\hline 2. & $\begin{array}{l}\text { ARAÚJO; OLIVEIRA; } \\
\text { FREITAS, } 2020\end{array}$ & $\begin{array}{l}\text { Em defesa do Sistema } \\
\text { Único de Saúde no } \\
\text { contexto da pandemia } \\
\text { por SARS-CoV-2 }\end{array}$ & $\begin{array}{l}\text { Discutir as condições } \\
\text { político-estruturais de } \\
\text { efetivação do SUS no } \\
\text { enfrentamento da pandemia } \\
\text { por SARS-CoV-2 }\end{array}$ \\
\hline 3. & BARBOSA, 2020 & $\begin{array}{l}\text { Estado nutricional e o } \\
\text { enfrentamento da } \\
\text { COVID-19: reflexões } \\
\text { para a prática de } \\
\text { enfermagem }\end{array}$ & $\begin{array}{l}\text { Reforçar a importância de se } \\
\text { atentar para da } \\
\text { monitoramento da } \\
\text { alimentação e iniciação da } \\
\text { terapia nutricional tão logo se } \\
\text { faça necessário. }\end{array}$ \\
\hline 4. & BARBOSA et al, 2020 & $\begin{array}{l}\text { Fatores de estresse } \\
\text { nos profissionais de } \\
\text { enfermagem no } \\
\text { combate à pandemia }\end{array}$ & $\begin{array}{l}\text { Identificar os principais } \\
\text { efeitos psicológicos da } \\
\text { pandemia da COVID-19 nos } \\
\text { profissionais de }\end{array}$ \\
\hline
\end{tabular}




\begin{tabular}{|c|c|c|c|}
\hline & & $\begin{array}{l}\text { da COVID-19: Síntese } \\
\text { de Evidências }\end{array}$ & $\begin{array}{l}\text { enfermagem; Os fatores } \\
\text { capazes de gerar estresse } \\
\text { psicológico nos professionais } \\
\text { de enfermagem; e as } \\
\text { estratégias de coping para o } \\
\text { combate ao estresse } \\
\text { emocional. }\end{array}$ \\
\hline 5. & BARRETO et al, 2020 & $\begin{array}{l}\text { O Repercussões da } \\
\text { pandemia de COVID- } \\
19 \text { na violência laboral } \\
\text { institucional aos } \\
\text { profissionais de } \\
\text { enfermagem }\end{array}$ & $\begin{array}{l}\text { Discutir sobre a violência } \\
\text { laboral institucional aos } \\
\text { profissionais de enfermagem } \\
\text { frente a pandemia de } \\
\text { COVID-19 }\end{array}$ \\
\hline 6. & BORGES et al, 2020 & $\begin{array}{l}\text { Enfermagem Militar na } \\
\text { "Operação Regresso } \\
\text { ao Brasil”: } \\
\text { aevacuação } \\
\text { pandemia } \\
\text { Coronavirus }\end{array}$ & $\begin{array}{l}\text { Descrever a experiência da } \\
\text { enfermagem militar na } \\
\text { Operação Regresso ao Brasil } \\
\text { em uma evacuação } \\
\text { aeromédica }\end{array}$ \\
\hline 7. & $\begin{array}{l}\text { CAVALCANTE; } \\
\text { SOUSA; DIAS, } 2020\end{array}$ & $\begin{array}{lr}\text { Consulta } & \text { de } \\
\text { Enfermagem aos } \\
\text { casos suspeitos de } \\
\text { COVID } \quad-19, \quad \text { na } \\
\text { Atenção Primária a } \\
\text { Saúde. }\end{array}$ & $\begin{array}{l}\text { Desenvolver uma revisão } \\
\text { sistemática sobre a Consulta } \\
\text { de Enfermagem aos casos } \\
\text { suspeitos de COVID -19, na } \\
\text { Atenção Primária a Saúde }\end{array}$ \\
\hline 8. & COSTA, 2020 & $\begin{array}{l}\text { Os desafios do } \\
\text { profissional de } \\
\text { enfermagem mediante } \\
\text { a COVID-19 }\end{array}$ & $\begin{array}{l}\text { Mostrar os obstáculos } \\
\text { significativos e simbólicos } \\
\text { impostos pela Covid-19 à } \\
\text { saúde da Enfermagem }\end{array}$ \\
\hline
\end{tabular}


9. COSTA et al, 2020

Ensino

enfermagem tempos de COVID-19: pandêmica

como se reinventar

nesse contexto

10. CRUZ et al, 2020

COVID-19: de Refletir sobre os dilemas da

em educação anteriores à crise

Emergência

e trabalho em profissionais Impactos na Saúde e que, hoje, atuam na linha de no Trabalho frente do combate à pandemia da COVID-19.

11. DAL'BOSCOI et al, A saúde mental da Identificar a prevalência e 2020

enfermagem

no fatores associados

enfrentamento

da ansiedade e depressão em COVID-19 em um profissionais de enfermagem hospital universitário que atuam no enfrentamento regional da COVID-19 em hospital universitário

12. FORTE; PIRES, 2020

\section{enter} meios

comunicação

apelos

da conhecer e analisar os tempos nos apelos da Enfermagem nas de mídias sociais durante a

Coronavirus.

13. FRANZOI;

Atuação

de Refletir sobre a atuação de

CAUDURO, 2020

estudantes de estudantes de graduação em enfermagem na enfermagem durante a pandemia de Covid- pandemia de Covid-19 19.

14. FREITAS; ALVES; Medidas de prevenção Identificar junto à literatura as GAÍVA, 2020 e controle de infecção medidas de prevenção e neonatal 


\begin{tabular}{|c|c|c|c|}
\hline & & $\begin{array}{l}\text { por COVID-19: revisão } \\
\text { de escopo }\end{array}$ & $\begin{array}{l}\text { controle de infecção neonatal } \\
\text { por COVID-19. }\end{array}$ \\
\hline 15. & $\begin{array}{l}\text { GALLASCHI et al, } \\
2020\end{array}$ & $\begin{array}{l}\text { Prevenção } \\
\text { relacionada à } \\
\text { exposição } \\
\text { ocupacional do } \\
\text { profissional de saúde } \\
\text { no cenário de COVID- } \\
19\end{array}$ & $\begin{array}{l}\text { descrever as principais } \\
\text { recomendações sobre ações } \\
\text { de prevenção de contágio } \\
\text { relacionadas à exposição } \\
\text { ocupacional dos profissionais } \\
\text { de saúde atuantes frente à } \\
\text { COVID-19, disponíveis até } \\
\text { março de } 2020\end{array}$ \\
\hline 16. & $\begin{array}{l}\text { HAMMERSCHMIDT; } \\
\text { BONATELLI; } \\
\text { CARVALHO, } 2020\end{array}$ & $\begin{array}{l}\text { Caminho da } \\
\text { esperança nas } \\
\text { relações envolvendo } \\
\text { os idosos: olhar da } \\
\text { complexidade sob } \\
\text { pandemia do covid-19 }\end{array}$ & $\begin{array}{l}\text { Refletir as relações } \\
\text { envolvendo os idosos } \\
\text { durante a pandemia COVID- } \\
19 \text { sob o olhar da } \\
\text { complexidade, vislumbrando } \\
\text { caminho da esperança. }\end{array}$ \\
\hline 17. & $\begin{array}{l}\text { HUMEREZ; } \\
\text { SILVA, } 2020\end{array}$ & $\begin{array}{l}\text { Saúde mental dos } \\
\text { profissionais de } \\
\text { enfermagem do Brasil } \\
\text { no contexto da } \\
\text { pandemia Covid-19: } \\
\text { ação do Conselho } \\
\text { Federal de } \\
\text { Enfermagem }\end{array}$ & $\begin{array}{l}\text { Refletir sobre a saúde mental } \\
\text { dos profissionais de } \\
\text { enfermagem brasileiros no } \\
\text { contexto da pandemia } \\
\text { COVID-19 }\end{array}$ \\
\hline 18. & LOURENÇÃO, 2020 & $\begin{array}{l}\text { A COVID-19 e os } \\
\text { desafios para o } \\
\text { sistema e os } \\
\text { profissionais de saúde }\end{array}$ & $\begin{array}{l}\text { Monitorando junto com o } \\
\text { COFEN as condições de } \\
\text { trabalho dos profissionais e } \\
\text { exigindorcondições } \\
\text { adequadas e seguras, com } \\
\text { fornecimento } \\
\text { Equipamentos de Proteção }\end{array}$ \\
\hline
\end{tabular}




\begin{tabular}{|c|c|c|c|}
\hline & & & $\begin{array}{l}\text { de Individual, } \\
\text { dimensionamento adequado } \\
\text { dos trabalhadores e } \\
\text { afastamento daqueles que se } \\
\text { encontram nos grupos de } \\
\text { risco. }\end{array}$ \\
\hline 19. & MEDEIROS, 2020 & $\begin{array}{l}\text { A luta dos } \\
\text { profissionais de saúde } \\
\text { no enfrentamento da } \\
\text { COVID-19 }\end{array}$ & Entender melhor o COVID-19 \\
\hline 20. & MEDVED et al, 2020 & $\begin{array}{l}\text { Atuação do } \\
\text { Enfermeiro Residente } \\
\text { na Testagem Rápida } \\
\text { para COVID-19: um } \\
\text { relato de experiência }\end{array}$ & $\begin{array}{l}\text { Relatar a experiência } \\
\text { observada por residente de } \\
\text { enfermagem nas testagens } \\
\text { do modelo drive-thru } \\
\text { acontecidas em Brasília- DF } \\
\text { no mês de abril de } 2020\end{array}$ \\
\hline 21. & MENESES, 2020 & $\begin{array}{l}\text { Gerenciamento } \\
\text { Emergencial de } \\
\text { Recursos da Atenção } \\
\text { Primária a Saúde no } \\
\text { Enfrentamento à } \\
\text { Pandemia da COVID- } \\
19\end{array}$ & $\begin{array}{l}\text { Apresentar a contribuição da } \\
\text { atenção primária à saúde } \\
\text { paulistana no gerenciamento } \\
\text { emergencial de recursos } \\
\text { para enfrentamento à } \\
\text { pandemia de Coronavírus. }\end{array}$ \\
\hline 22. & $\begin{array}{l}\text { MINISTÉRIO } \\
\text { SAÚDE, } 2020\end{array}$ & $\begin{array}{l}\text { Orientações para } \\
\text { manejo de pacientes } \\
\text { com COVID-19. }\end{array}$ & $\begin{array}{l}\text { Oferecer orientações aos } \\
\text { gestores e profissionais de } \\
\text { saúde sobre o manejo dos } \\
\text { pacientes com suspeita ou } \\
\text { confirmação de COVID-19 } \\
\text { nos diversos pontos da Rede } \\
\text { de Atenção à Saúde, com } \\
\text { foco em uma assistência }\end{array}$ \\
\hline
\end{tabular}




\begin{tabular}{|c|c|c|c|}
\hline & & & $\begin{array}{l}\text { qualificada e em tempo } \\
\text { oportuno, desde as pessoas } \\
\text { assintomáticas até aquelas } \\
\text { com manifestações mais } \\
\text { severas da doença. }\end{array}$ \\
\hline 23. & MIRANDA et al, 2020 & $\begin{array}{l}\text { Condições de trabalho } \\
\text { e o impacto na saúde } \\
\text { dos profissionais de } \\
\text { enfermagem frente a } \\
\text { Covid-19 }\end{array}$ & $\begin{array}{l}\text { Refletir sobre as condições } \\
\text { de trabalho dos profissionais } \\
\text { de enfermagem no } \\
\text { enfrentamento ao novo } \\
\text { Coronavírus e apontar o } \\
\text { impacto na vida desses } \\
\text { profissionais em meio à } \\
\text { pandemia }\end{array}$ \\
\hline 24. & MOITINHO et al, 2020 & $\begin{array}{l}\text { Lesão renal aguda } \\
\text { pelo vírus SARS-COV- } \\
2 \text { em pacientes com } \\
\text { COVID-19: revisão } \\
\text { integrativa }\end{array}$ & $\begin{array}{l}\text { Avaliar as evidências } \\
\text { científicas sobre Lesão Renal } \\
\text { Aguda pela SARS-CoV-2 em } \\
\text { pacientes com COVID-19. }\end{array}$ \\
\hline 25. & $\begin{array}{l}\text { MOURA; LOPEZ; } \\
\text { SOARES, } 2020\end{array}$ & $\begin{array}{l}\text { Informações } \\
\text { epidemiológicas sobre } \\
\text { a COVID-19: } \\
\text { Influência da } \\
\text { cibercultura no } \\
\text { engajamento popular } \\
\text { às medidas de } \\
\text { controle }\end{array}$ & $\begin{array}{l}\text { Analisar a comunicação de } \\
\text { informações epidemiológicas } \\
\text { para a adesão da população } \\
\text { às medidas de controle, na } \\
\text { perspectiva de Pierre Levy e } \\
\text { Littlejohn. }\end{array}$ \\
\hline 26. & NETO et al, 2020 & $\begin{array}{lr}\text { COVID-19 } & \text { e } \\
\text { tecnologia } & \text { digital: } \\
\text { aplicativos } & \text { móveis } \\
\text { disponíveis } & \text { para }\end{array}$ & $\begin{array}{l}\text { Descrever aplicativos móveis } \\
\text { sobre Covid-19 disponíveis } \\
\text { para download em } \\
\text { smartphones. }\end{array}$ \\
\hline
\end{tabular}




\begin{tabular}{|c|c|c|c|}
\hline & & $\begin{array}{l}\text { download } \quad \text { em } \\
\text { smartphones }\end{array}$ & \\
\hline 27. & $\begin{array}{l}\text { NETO; } \\
\text { BORTOLUZZI; } \\
\text { FREITAS, } \\
2020\end{array}$ & $\begin{array}{l}\text { Equipamentos de } \\
\text { proteção individual } \\
\text { para prevenção de } \\
\text { infecção por SARS- } \\
\text { COV-2 }\end{array}$ & $\begin{array}{l}\text { Discutir sobre as indicações } \\
\text { relacionadas ao uso dos } \\
\text { Equipamentos de Proteção } \\
\text { Individual (EPI) por parte dos } \\
\text { profissionais que atuam na } \\
\text { assistência prestada aos } \\
\text { pacientes contaminados ou } \\
\text { suspeitos de Coronavírus, a } \\
\text { fim de reduzir ou impedir o } \\
\text { aumento dos casos nestes } \\
\text { profissionais. }\end{array}$ \\
\hline 28. & OLIVEIRA, 2020 & $\begin{array}{lr}\text { Desafios } & \text { da } \\
\text { enfermagem frente ao } \\
\text { enfrentamento da } \\
\text { pandemia } \\
\text { COVID19 }\end{array}$ & $\begin{array}{l}\text { Realizar uma reflexão sobre } \\
\text { a Enfermagem, sua atuação, } \\
\text { contribuição } \\
\text { reconhecimento, } \\
\text { especialmente no ano a ela } \\
\text { dedicado, pela celebração do } \\
\text { bicentenário do aniversário } \\
\text { de Florence Nightingale, } \\
\text { fundadora da Enfermagem } \\
\text { Moderna. }\end{array}$ \\
\hline 29. & OLIVEIRA et al, 2020 & $\begin{array}{l}\text { Equipamento de } \\
\text { Proteção Individual na } \\
\text { pandemia por } \\
\text { Coronavírus: } \\
\text { treinamento com } \\
\text { Prática Deliberada em } \\
\text { Ciclos Rápidos }\end{array}$ & $\begin{array}{l}\text { discutir a aplicação da } \\
\text { Prática Deliberada em Ciclos } \\
\text { Rápidos para o treinamento } \\
\text { de paramentação e } \\
\text { desparamentação no } \\
\text { contexto da COVID-19 e } \\
\text { estruturar um guia prático }\end{array}$ \\
\hline
\end{tabular}




\begin{tabular}{|c|c|c|c|}
\hline & & & $\begin{array}{l}\text { para a aplicação nesta } \\
\text { conjuntura }\end{array}$ \\
\hline 30. & $\begin{array}{l}\text { OLIVEIRA; LUCAS; } \\
\text { IQUIAPAZA, } 2020 .\end{array}$ & $\begin{array}{l}\text { O que a pandemia da } \\
\text { Covid-19 tem nos } \\
\text { ensinado sobre } \\
\text { adoção de medidas de } \\
\text { precaução? }\end{array}$ & $\begin{array}{l}\text { Analisar a pandemia da } \\
\text { Covid-19 e o que temos (re) } \\
\text { aprendido com a experiência } \\
\text { mundial para adoção das } \\
\text { medidas de prevenção } \\
\text { preconizadas pela OMS bem } \\
\text { como o panorama } \\
\text { epidemiológico no mundo, na } \\
\text { América Latina e no Brasil. }\end{array}$ \\
\hline 31. & $\begin{array}{l}\text { PARREIRA et al, } \\
2020\end{array}$ & $\begin{array}{l}\text { Cuidados de } \\
\text { enfermagem em } \\
\text { tempos de pandemia: } \\
\text { Umaralidade } \\
\text { hospitalar }\end{array}$ & $\begin{array}{l}\text { Descrever as alterações } \\
\text { realizadas na CUF Infante } \\
\text { Santo Hospital para } \\
\text { responder à pandemia e o } \\
\text { papel das equipas de } \\
\text { enfermagem neste contexto, } \\
\text { sobretudo a nível de } \\
\text { internamento hospitalar }\end{array}$ \\
\hline 32. & $\begin{array}{l}\text { PAULA et al, } 2020 \\
\text { (a) }\end{array}$ & $\begin{array}{l}\text { Higiene das mãos em } \\
\text { setores de alta } \\
\text { complexidade como } \\
\text { elemento integrador } \\
\text { no combate do Sars- } \\
\text { CoV-2. }\end{array}$ & $\begin{array}{l}\text { realizar um diagnóstico } \\
\text { situacional } \\
\text { comportamento } \\
\text { profissionais de saúde } \\
\text { quanto às práticas de } \\
\text { higienização das mãos em } \\
\text { setores de alta } \\
\text { complexidade. }\end{array}$ \\
\hline 33. & $\begin{array}{l}\text { PAULA et al, } 2020 \\
\text { (b) }\end{array}$ & $\begin{array}{l}\text { A enfermagem frente } \\
\text { ao processo de morte } \\
\text { e morrer: uma reflexão }\end{array}$ & $\begin{array}{l}\text { Refletir sobre os desafios da } \\
\text { enfermagem diante do } \\
\text { processo de morte e morrer }\end{array}$ \\
\hline
\end{tabular}




\begin{tabular}{|c|c|c|c|}
\hline & & $\begin{array}{l}\text { em tempos de } \\
\text { Coronavírus }\end{array}$ & $\begin{array}{l}\text { em face a pandemia por } \\
\text { Coronavírus. }\end{array}$ \\
\hline 34. & $\begin{array}{l}\text { PORTUGAL et al, } \\
2020\end{array}$ & $\begin{array}{l}\text { Percepção do impacto } \\
\text { emocional da equipe } \\
\text { de enfermagem diante } \\
\text { da pandemia de } \\
\text { COVID-19: relato de } \\
\text { experiência. }\end{array}$ & $\begin{array}{l}\text { Relatar a percepção da } \\
\text { equipe de enfermagem de } \\
\text { um hospital em um município } \\
\text { no interior do estado do } \\
\text { Amazonas, diante da } \\
\text { pandemia de Coronavírus, } \\
\text { expondo os principais medos } \\
\text { e anseios frente as } \\
\text { incertezas do novo cenário } \\
\text { mundial e os desafios } \\
\text { vindouros. }\end{array}$ \\
\hline 35. & RAFAELI et al, 2020 & $\begin{array}{l}\text { Epidemiologia, } \\
\text { políticas públicas e } \\
\text { pandemia de Covid- } \\
\text { 19: o que esperar no } \\
\text { Brasil? }\end{array}$ & $\begin{array}{l}\text { Discutir as experiências e } \\
\text { contradições no controle da } \\
\text { pandemia de Covid- } 19 \text { sob a } \\
\text { perspectiva da epidemiologia } \\
\text { e das políticas públicas } \\
\text { brasileiras }\end{array}$ \\
\hline 36. & $\begin{array}{l}\text { RIBOLI; ARTHUR; } \\
\text { MANTOVANI, } 2020\end{array}$ & $\begin{array}{l}\text { No epicentro da } \\
\text { epidemia: um olhar } \\
\text { sobre a COVID-19 na } \\
\text { Itália }\end{array}$ & $\begin{array}{l}\text { Refletir sobre os impactos da } \\
\text { COVID-19 no norte da Itália, } \\
\text { epicentro europeu da } \\
\text { pandemia. }\end{array}$ \\
\hline 37. & ROCHA et al, 2020 & $\begin{array}{l}\text { Construindo o projeto } \\
\text { cuidadosamente: } \\
\text { reflexão sobre a saúde } \\
\text { mental dos } \\
\text { graduandos de } \\
\text { Enfermagem frente ao } \\
\text { COVID-19 }\end{array}$ & $\begin{array}{l}\text { apontar a sua inserção na } \\
\text { prática, bem como, retratar a } \\
\text { importância de um projeto } \\
\text { desta magnitude no cuidado } \\
\text { à saúde psíquica dos } \\
\text { acadêmicos de enfermagem, } \\
\text { principalmente no contexto }\end{array}$ \\
\hline
\end{tabular}


atual de isolamento social pela pandemia de COVID-19

38. RODRIGUES; SILVA, Gestão da pandemia Descrever a experiência da 2020 Coronavírus em um gestão para 0 atendimento hospital: relato de de paciente confirmado ou experiência com suspeita de Coronavírus profissional em um hospital da região metropolitana de Porto Alegre

39. RODRIGUES et al, Medidas de contenção Descrever a experiência da 2020 à COVID-19 adotadas enfermagem, na adoção de em serviço de medidas de contenção, no transplante de medula atendimento de pacientes óssea. submetidos ao transplante de células-tronco hematopoiéticas para evitar a COVID-19

40. SAIDELI et al, 2020 Intervenções em refletir sobre as saúde mental para intervenções/ações de profissionais de saúde cuidado em saúde mental frente a pandemia de voltados aos profissionais da Coronavírus saúde que prestam assistência ao paciente suspeito ou diagnosticado com COVID-19

41. SILVA, 2020

Enfermagem

no Mostrar relevância social combate à pandemia sobre o trabalho em saúde da COVID-19

42. SILVA et al, 2020 (a) Planejamento Refletir sobre o planejamento organizacional no organizacional no contexto contexto de pandemia da pandemia por COVID-19 
por

COVID-19: e as implicações para a implicações para a gestão em enfermagem.

gestão em

enfermagem

43. SILVA et al, 2020 (b) Anos de vida perdidos Estimar a carga da doença ajustados por atribuível a infecção pelo incapacidade (DALY) COVID-19 entre os entre os profissionais profissionais de de enfermagem enfermagem, estimar os devido a infecção pelo anos de vida perdidos devido COVID-19 no Brasil.

a mortalidade prematura (YLL), estimar os anos perdidos por incapacidade (YLD) e estimar os anos de vida ajustados por incapacidade (DALY).

44.

Pandemia de COVID- Descrever as

SOARES et al, 2020

19 e o uso racional de recomendações sobre o uso equipamentos de racional e seguro dos proteção individual equipamentos de proteção individual (EPI) na cadeia assistencial de pessoas com suspeita ou confirmação de contaminação pelo novo Coronavírus

45. SOUSA et al, 2020

\begin{tabular}{l|llr} 
Estimação e predição & Estimar a taxa & de \\
dos casos de COVID- & transmissão, & o pico \\
19 nas metrópoles & epidemiológico e óbitos pelo \\
brasileiras. & novo Coronavírus.
\end{tabular}

46. SOUZA; SOUZA, Enfermagem brasileira Discutir desafios da 2020 na linha de frente Enfermagem Brasileira na 


\begin{tabular}{|c|c|c|c|}
\hline & & $\begin{array}{l}\text { contra o novo } \\
\text { Coronavírus: } \\
\text { cuidará de de } \\
\text { cuida? }\end{array}$ & $\begin{array}{l}\text { linha de frente contra o novo } \\
\text { Coronavírus. }\end{array}$ \\
\hline 47. & SOUZA et al, 2020 & $\begin{array}{l}\text { Estágio curricular } \\
\text { supervisionado em } \\
\text { enfermagem durante a } \\
\text { pandemia de } \\
\text { Coronavírus: } \\
\text { experiências na } \\
\text { atenção básica. }\end{array}$ & $\begin{array}{l}\text { Relatar as experiências de } \\
\text { estudantes de enfermagem } \\
\text { durante o estágio curricular } \\
\text { supervisionado na atenção } \\
\text { básica no cenário da } \\
\text { pandemia de Coronavírus. }\end{array}$ \\
\hline 48. & $\begin{array}{l}\text { TAETS; BARBOSA; } \\
\text { TAETS, } \\
2020\end{array}$ & $\begin{array}{l}\text { Padrões funcionais de } \\
\text { saúde em adultos com } \\
\text { COVID-19 na terapia } \\
\text { intensiva: } \\
\text { fundamentação aos } \\
\text { diagnósticos de } \\
\text { enfermagem. }\end{array}$ & $\begin{array}{l}\text { Identificar os principais } \\
\text { diagnósticos de enfermagem } \\
\text { em pacientes adultos } \\
\text { confirmados com o novo } \\
\text { Coronavírus internados em } \\
\text { unidades de terapia intensiva } \\
\text { à luz dos padrões funcionais } \\
\text { de saúde. }\end{array}$ \\
\hline 49. & $\begin{array}{l}\text { TAMINATO et al, } \\
2020\end{array}$ & $\begin{array}{l}\text { Máscaras de tecido na } \\
\text { contenção de } \\
\text { gotículas respiratórias } \\
\text { - revisão sistemática }\end{array}$ & $\begin{array}{l}\text { Identificar, avaliar } \\
\text { sistematicamente } \\
\text { sumarizar as melhores } \\
\text { evidências científicas } \\
\text { disponíveis sobre a eficácia e } \\
\text { a segurança das máscaras } \\
\text { de tecido para a comunidade. }\end{array}$ \\
\hline 50. & TAVARES, 2020 & $\begin{array}{l}\text { Dimensões do cuidado } \\
\text { na perspectiva da } \\
\text { espiritualidade } \\
\text { durante a pandemia }\end{array}$ & $\begin{array}{l}\text { Apresentar uma reflexão, } \\
\text { nesse momento ímpar de } \\
\text { crise sanitária, a pandemia } \\
\text { pela COVID-19 }\end{array}$ \\
\hline
\end{tabular}




\section{pelo novo Coronavírus}

(COVID-19)

51. TONIN et al, 2020

\begin{tabular}{ll|l} 
Recomendações em & Propor recomendações para \\
tempos de COVID-19: & a prática de enfermagem \\
\hline um olhar para o & domiciliar no contexto da \\
cuidado domiciliar. & COVID-19.
\end{tabular}

Fonte: Elaborado pelos autores (2020).

\section{DISCUSSÃO}

\section{AS DIFICULDADES DA ENFERMAGEM DURANTE O COMBATE DO COVID-19}

Os resultados dos estudos mostram que o principal protagonismo da enfermagem durante o combate da COVID-19, foi o processo de humanização, que além de ter o papel fundamental na criação de diversas campanhas educativas e criação de aplicativos, destacou-se como força expressiva e completamente necessária no enfrentamento do novo vírus (NETO et al, 2020).

Nos dias atuais a enfermagem tem diversos desafios durante o combate do Covid-19, pois a pandemia está progredindo e aumentando o índice de infecção e subitamente vem transformando a rotina dos hospitais. A enfermagem vivencia constantemente a escassez de equipamentos médicos hospitalares e profissionais de enfermagem, jornadas de trabalhos exaustivas por vezes ultrapassando os limites do cansaço e segurança ocupacional (COSTA, 2020; LOURENÇÃO, 2020; OLIVEIRA, 2020; PAULA et al, 2020 (b); PORTUGAL et al, 2020).

Segundo autores, o momento é difícil para enfermagem, pois presencia uma guerra contra uma ameaça invisível jamais prevista pelas as autoridades mundiais de saúde. A falta de insumos e equipamentos para a proteção individual, leva os profissionais reutilizar os EPIS, atentando contra a segurança dos profissionais. O conselho nacional de enfermagem no Brasil teve 3,6 mil denúncias sobre a qualidade e falta de 
EPIS como: Luvas, aventais e máscaras, está situação trás bastante preocupação para os conselhos regionais de enfermagem (COREN) (NETO; BORTOLUZZI; FREITAS, 2020; SOARES et al, 2020).

Estudos mostram que os profissionais da enfermagem mesmo que estejam paramentados não consegue deixar as longas jornadas de trabalho, devido o número insuficiente de técnicos e enfermeiros no serviço de saúde. Relatos como: lesões por uso prolongado de máscara, impossibilidade de remoção de máscara, escassez de luvas e aventais, falta de treinamento para prestar os cuidados de enfermagem ao paciente com COVID-19 e na paramentação e desparamentação de EPI. Tudo isso gera insegurança do iminente risco de se-contaminar e contaminar sua família (NETO; BORTOLUZZI; FREITAS, 2020; OLIVEIRA et al, 2020; SOARES et al, 2020).

É evidente que a enfermagem está vivenciando um dilema moral e ético: prestar assistência de enfermagem sem utilizar EPI da forma adequada colocando, entretanto, a própria vida em risco e dos seus familiares, porém ao negar atendimento para o paciente em estado de emergência ou urgência, podem ser responsabilizados conforme o Art.135 do código penal Brasileiro (MINISTÉRIO DA SAÚDE, 2020; MIRANDA et al, 2020; NETO; BORTOLUZZI; FREITAS, 2020; OLIVEIRA et al, 2020; SOARES et al, 2020).

Em meio a esta situação, estudos descrevem que os profissionais da enfermagem são uma das categorias mais afetadas pelo o estresse durante o combate da COVID19. Nesse contexto, a organização mundial da saúde (OMS), lançou uma cartilha para orientar os profissionais da saúde para lidar com a rotina estressante proporcionada pelo o CORONAVIRUS, combater agravos severos de saúde mental e prevenir doenças como a síndrome de Burnout (BARBOSA et al, 2020; RODRIGUES; SILVA, 2020; SILVA, 2020).

\section{O PROTAGONISMO DA ENFERMAGEM NO COMBATE DO COVID-19}

A pandemia do COVID-19 traz mudanças significativas para a enfermagem, devido à sobrecarga de trabalho, aos altos índices de transmissão do vírus e a falta de EPI e 
treinamento. Esta experiência vem sendo vivenciada tanto na rede pública quanto privada ao nível mundial (BARRETO et al, 2020; HUMEREZ; OHL; SILVA, 2020; LOURENÇÃO, 2020; MIRANDA et al, 2020; MOURA; LOPEZ; SOARES, 2020; OLIVEIRA, 2020; PARREIRA et al, 2020; RIBOLI; ARTHUR; MANTOVANI, 2020).

Os obstáculos que foram enfrentados pela a enfermagem durante a pandemia do Covid-19 pode ser categorizada em limites, insuficiências e carências. Os profissionais da enfermagem se depararam constantemente com a falta de equipamentos de proteção individual (EPI), insumos básicos, leitos de UTI e estresse ocasionado pelo o esgotamento físico e mental, além do medo da contaminação (COSTA, 2020; NETO; BORTOLUZZI; FREITAS, 2020; OLIVEIRA et al, 2020; SOARES et al, 2020).

Entretanto, mesmo neste momento difícil a enfermagem rapidamente sofreu uma metamorfose e revelou a sua extrema importância no combate do COVID-19 mostrando grande protagonismo. Segundo Parreira et al (2020) criou as ações e estratégias básicas de controle de infecção como:

- Treinamento sobre utilização de equipamentos de proteção individual, ensinando o profissional de saúde realizar a sequência de paramentação e desparamentação;

- Reforçando e salientando a importância das higienes das mãos com solução antisséptica;

- Ensinando a etiqueta respiratória para a equipe multiprofissional e pacientes;

- Elaborou a criação de planos para a hotelaria hospitalar, por meio de treinamento da equipe multiprofissional como; descontaminação de equipamento clínico, recolhimento de roupas e resíduos hospitalares.

Outros autores afirmam que, após a criação de diversas estratégias, o principal papel desempenhado pela a enfermagem na pandemia por COVID-19 foi o controle e prevenção de infecção, dentre das quais destaca-se a higienização das mãos e cuidados com apresentação pessoal como; Barba feita ou aparada, unhas limpas e curtas, sapatos que apresentem a sola de borracha para evitar quedas e ruídos. Para 
iniciar a jornada de trabalho o profissional de saúde deve realizar os preparativos iniciais; retirando adornos (alianças, correntes, anéis, relógios e brincos etc.), remover utensílios pessoais e utilização de EPI de forma correta (NETO; BORTOLUZZI; FREITAS, 2020; SOARES et al, 2020)

Ainda teve destaque no acolhimento com enfoque educativo para a população, sanando dúvidas e reforçando as orientações individuais de saúde, por meio de campanhas educativas em mídias sociais visando conscientizar a população sobre os protocolos de prevenção do COVID-19, sintomas, grupos de risco e aspectos básicos de coletas de exames para Coronavírus (COSTA et al, 2020; MINISTÉRIO DA SAÚDE, 2020; MEDVED et al, 2020).

Para auxiliar na prevenção da contaminação por COVID-19 o Conselho federal de Enfermagem (COFEN) junto com o ministério saúde, desenvolveram um grande número de aplicativos sobre o COVID-19, que teve bastante engajamento de governos estaduais e federais no combate da pandemia, disponibilizando conteúdo educativo para a população, além de ações preventivas, levantamento de dados e otimização do tempo nas atividades assistenciais (ABEN, 2020; COFEN, 2020; MINISTÉRIO DA SAÚDE, 2020; NETO et al, 2020).

Segundo BORGES et al (2020) a enfermagem militar brasileira teve grande importância na Operação Regresso ao Brasil em uma Evacuação Aeromédica (EVAM). A missão EVAM foi realizada para repatriar brasileiros que estavam localizados em WUHAM no epicentro da pandemia de Corona vírus, também teve grande importância no treinamento e atendimento com segurança e qualidade dos pacientes que foram repatriados. Além de se desempenhar de forma extenuante e inédita na história da enfermagem no transporte aeromédico.

\section{CONSIDERAÇÕES FINAIS}

Em meio a guerra contra o novo Coronavírus, os profissionais não mediram esforços para orientar a população sobre os cuidados na prevenção do COVID-19, assumindo assim, um grande papel no combate do COVID-19. Neste momento a enfermagem 
deve mostrar seu empoderamento, pois suas ações de proteção da saúde da população estão salvando vidas diariamente.

Sabendo disso, cabe a enfermagem mostrar o conhecimento sobre as medidas de prevenção e segurança para assegurar o atendimento digno, mesmo em frente a um grande desafio que foi proporcionado pela a epidemia do COVID-19. Destacou-se ainda, que os profissionais da enfermagem com todos os desafios como: jornada de trabalho exaustiva, estresse, insegurança e medo, ficam realizados e felizes quando o paciente consegue sair recuperado.

Foi possível evidenciar que o principal protagonismo da enfermagem foi realizar o processo de humanização em um ambiente completamente hostil, cercado de medo e insegurança, trazendo discussões significativas para o conhecimento dos valores éticos e morais na atuação da Enfermagem diante deste cenário atual desafiador. Além da humanização, percebe-se que a enfermagem teve papel fundamental no combate do novo covid-19, realizando diversas campanhas de educativas e criação de aplicativos, se apresentando também como força expressiva e completamente necessária no enfrentamento do novo COVID-19.

\section{REFERÊNCIAS BIBLIOGRÁFICAS}

ASSOCIAÇÃO BRASILEIRA DE ENFERMAGEM (ABEn). Nota da ABEN Nacional em relação à Ação Estratégia "O Brasil Conta Comigo". 2020 [acesso em 19 setembro 2020]; Disponível em: http://www.abennacional.org.br/site/wpcontent/uploads/2020/04/Nota-Aben-educacao2.pdf.

AMITRANO, C; MAGALHÃES, L. C. G; SILVA.M, S. Medidas de enfrentamento dos efeitos econômicos da pandemia COVID-19: panorama internacional e análise dos casos dos Estados Unidos, do Reino unido e da Espanha. (c) Instituto de Pesquisa Econômica Aplicada - IPEA 2019.

ARAÚJO, J. L; OLIVEIRA, K. K. D; FREITAS, R. J. M. Em defesa do Sistema Único de Saúde no contexto da pandemia por SARS-CoV-2. Rev Bras Enferm. 2020;73. 
BARBOSA, J. A. G. Estado nutricional e o enfrentamento da COVID-19: reflexões para a prática de enfermagem; Braz. J. Hea. Rev., Curitiba, v. 3, n. 3, p. 6671-6675 may./jun. 2020.

BARBOSA, D. J; et al. Fatores de estresse nos profissionais de enfermagem no combate à pandemia da COVID-19: Síntese de Evidências; Ciências Saúde 2020;31 Suppl 1:31-47.

BARRETO, F. A; et al. O Repercussões da pandemia de COVID-19 na violência laboral institucional aos profissionais de enfermagem. Universidade do Estado do Rio Grande do Norte (UERN). Departamento de Enfermagem, campus Pau dos Ferros, Rio Grande do Norte, Brasil. 2020

BORGES, L. L; et al. Enfermagem Militar na "Operação Regresso ao Brasil": evacuação aeromédica na pandemia do coronavírus. Rev Bras Enferm. 2020;73(Suppl 2): 1

CAVALCANTE, C. C. F. S; SOUSA, J. A. S; DIAS, A. M. A; Consulta de Enfermagem aos casos suspeitos de COVID -19, na Atenção Primária a Saúde. Revista da FAESF, vol. 4. Número especial COVID 19. Junho (2020) 34-40.

CESÁRIO, J. M. S; FLAUZINO, V. H. P; MEJIA, J. V; Metodologia científica: Principais tipos de pesquisas e suas caraterísticas. Revista Científica Multidisciplinar Núcleo do Conhecimento. Ano 05, Ed. 11, Vol. 05, pp. 23-33. Novembro de 2020.

CONSELHO FEDERAL DE ENFERMAGEM (COFEN). Cofen se manifesta sobre a Portaria 356 do MEC. 2020 [acesso em 19 setembro 2020]. Disponível em: http://www.cofen.gov.br/cofen-se-manifestasobre-a-portaria-356-domec_78941.html.

COSTA, D. M. Os desafios do profissional de enfermagem mediante a COVID-19; Gestão \& Tecnologia Faculdade Delta. Ano IX, V. 1 Edição 30 Jan/Jun 2020. 
COSTA, R; et al. Ensino de enfermagem em tempos de COVID-19: como se reinventar nesse contexto. Texto \& Contexto Enfermagem 2020, v. 29: e20200202.

CRUZ, R. M; et al. COVID-19: Emergência e impactos na saúde e no trabalho. Revista Psicologia: Organizações e Trabalho, 20(2), abr-jun. 2020, I-III.

DAL'BOSCO, E. B; et al. A saúde mental da enfermagem no enfrentamento da COVID-19 em um hospital universitário regional. Rev Bras Enferm. 2020;73(Suppl 2): 1

FORTE, E. C. N; PIRES, D. E. P. Os apelos da enfermagem nos meios de comunicação em tempos de coronavirus. Rev Bras Enferm. 2020;73

FRANZOI, M. A. H; CAUDURO, F. L. F; Atuação de estudantes de enfermagem na pandemia de Covid-19. Cogitare enferm. 25: e73491, 2020.

FREITAS, B. M. B. M; ALVES, M. D.S. M; GAÍVA, M.A. M; Medidas de prevenção e controle de infecção neonatal por COVID-19: revisão de escopo. Rev Bras Enferm. $2020 ; 73$

GALLASCHI, C. H; et al. Prevenção relacionada à exposição ocupacional do profissional de saúde no cenário de COVID-19. Rev enferm UERJ, Rio de Janeiro, 2020; 28: e49596.

HAMMERSCHMIDT, K. S. A; BONATELLI, L. C. S; CARVALHO, A. C. Caminho da esperança nas relações envolvendo os idosos: olhar da complexidade sob pandemia do covid-19. Texto \& Contexto Enfermagem 2020.

HUMEREZ, D. C; OHL, R. I. B; SILVA, M. C. N. Saúde mental dos profissionais de enfermagem do Brasil no contexto da pandemia Covid-19: ação do Conselho Federal de Enfermagem; Cogitare enferm. 25: e74115, 2020.

LOURENÇÃO, L. G. A COVID-19 e os desafios para o sistema e os profissionais de saúde; Revista do conselho de enfermagem PA. v. 11, n. 1 (2020). 
MEDEIROS, E. A. A luta dos profissionais de saúde no enfrentamento da COVID19. Acta Paul Enferm. 2020;33: e-EDT20200003.

MEDVED, I. V; et al. Atuação do Enfermeiro Residente na Testagem Rápida para COVID-19: um relato de experiência; v. 1 n. 2 (2020): Pandemia COVID-19.

MENESES, A. S. Gerenciamento Emergencial de Recursos da Atenção Primária a Saúde no Enfrentamento à Pandemia da COVID-19. SciELO Preprints, 2020.

MINISTÉRIO DA SAÚDE (BR). Secretaria de Vigilância em Saúde. Boletim Epidemiológico Especial n. 14. COE-COVID19. [Internet]. Brasília: Ministério da Saúde; 2020 [acesso em 19 setembro 2020]. Disponível em: https://portalarquivos.saude.gov.br/images/pdf/2020/April/27/2020-04-27-18-05h-

BEE14-Boletim-doCOE.pdf.

MIRANDA, F. M. A; et al. Condições de trabalho e o impacto na saúde dos profissionais de enfermagem frente a Covid-19. Cogitare enferm. 25: e72702, 2020.

MOITINHO. M. S; et al. Lesão renal aguda pelo vírus SARS-COV-2 em pacientes com COVID-19: revisão integrativa. Rev Bras Enferm. 2020;73(Suppl 2): 1

MOURA, E. C. C; LOPEZ, V; SOARES, S. S. Informações epidemiológicas sobre a COVID-19: Influência da cibercultura no engajamento popular às medidas de controle. Cogitare enferm. 25: e74566, 2020.

NETO, N. M. G; et al. Covid-19 e tecnologia digital: aplicativos móveis disponíveis para download em smartphones. Texto \& Contexto Enfermagem 2020, v. 29: e20200150

NETO, A. R. S; BORTOLUZZI, B. B; FREITAS, D. R. J; Equipamentos de proteção individual para prevenção de infecção por SARS-COV-2. J Manag Prim Health Care, 2020;12: e17 
OLIVEIRA, A. C; Desafios da enfermagem frente ao enfrentamento da pandemia da COVID19; Rev Min Enferm. 2020;24: e-1302

OLIVEIRA, H. C; et al. Equipamento de Proteção Individual na pandemia por coronavírus: treinamento com Prática Deliberada em Ciclos Rápidos. Rev Bras Enferm. 2020;73(Suppl 2): 1

OLIVEIRA, A. C; LUCAS, T. C; IQUIAPAZA, R. A. O que a pandemia da Covid-19 tem nos ensinado sobre adoção de medidas de precaução? Texto \& Contexto Enfermagem 2020, v. 29: e20200106.

PARREIRA, S. T. P; et al. Cuidados de Enfermagem em Tempos de Pandemia: Uma Realidade Hospitalar; Gazeta médica nํ2 vol. 7 · abril/junho 2020.

PAULA, D. G; et al (a). Higiene das mãos em setores de alta complexidade como elemento integrador no combate do Sars-CoV-2. Rev Bras Enferm. 2020;73(Suppl 2): 1

PAULA, G. S; et al (b). A enfermagem frente ao processo de morte e morrer: uma reflexão em tempos de Coronavírus. J. nurs. health. 2020;10(n.esp.): e20104018

PORTUGAL, J. K. A; et al. Percepção do impacto emocional da equipe de enfermagem diante da pandemia de COVID-19: relato de experiência; REAS/EJCH | Vol. Esp. 46 | e3794

RAFAELI, R. M. R; et al. Epidemiologia, políticas públicas e pandemia de Covid19: o que esperar no Brasil? Rev enferm UERJ, Rio de Janeiro, 2020; 28: e49570.

RIBOLI, E; ARTHUR, J. P; MANTOVANI, M. F. No epicentro da epidemia: um olhar sobre a Covid-19 na Itália. Cogitare enferm. 25: e72955, 2020

ROCHA, N. L; et al. Construindo o Projeto Cuidadosamente: reflexão sobre a saúde mental dos graduandos de Enfermagem frente ao COVID-19. Rev. Saúde Col. UEFS, Feira de Santana, Vol. 10: 13-17 (2020) 
RODRIGUES, N. H; SILVA, L. G. A; Gestão da pandemia Coronavírus em um hospital: relato de experiência profissional. J. nurs. health. 2020;10(n.esp): e20104004.

RODRIGUES, J. Á. P; et al. Medidas de contenção à COVID-19 adotadas em serviço de transplante de medula óssea. Rev Bras Enferm. 2020;73

SAIDELI, M. G. B; et al. Intervenções em saúde mental para profissionais de saúde frente a pandemia de Coronavírus. Rev enferm UERJ, Rio de Janeiro, 2020; 28: e49923.

SILVA, F. V. Enfermagem no combate à pandemia da COVID-19; Acta Paul Enferm. 2020; 33:1-11.

SILVA, J. M. A. V; et al (a). Planejamento organizacional no contexto de pandemia por COVID-19: implicações para a gestão em enfermagem; Journal Health NPEPS. 2020 jan-jun; 5(1): e4626.

SILVA, R. C. L; et al (b). Anos de vida perdidos ajustados por incapacidade (DALY) entre os profissionais de enfermagem devido a infecção pelo COVID-19 no Brasil; Universidade Federal do Estado do Rio de Janeiro - UNIRIO. Rio de Janeiro, RJ, Brasil 2020.

SOARES, S. S. S; et al. Pandemia de COVID-19 e o uso racional de equipamentos de proteção individual. Rev enferm UERJ, Rio de Janeiro, 2020; 28: e50360.

SOUSA, G. J. B; et al. Estimação e predição dos casos de COVID-19 nas metrópoles brasileiras. Rev. Latino-Am. Enfermagem. 2020;28: e3345.

SOUZA, L. P. S; SOUZA, A. G. Enfermagem brasileira na linha de frente contra o novo Coronavírus: quem cuidará de quem cuida? J. nurs. health. 2020;10(n.esp.): e20104005 
SOUZA, L. B; et al. Estágio curricular supervisionado em enfermagem durante a pandemia de Coronavírus: experiências na atenção básica. J. nurs. health. 2020;10(n.esp.): e20104017.

TAETS, G. G. C; BARBOSA, J. E. S; TAETS, C. M. C. Padrões funcionais de saúde em adultos com COVID-19 na terapia intensiva: fundamentação aos diagnósticos de enfermagem. Universidade Federal do Rio de Janeiro, Campus Macaé 2020.

TAMINATO, M; et al. Máscaras de tecido na contenção de gotículas respiratórias - Revisão sistemática. Acta Paul. Enferm. 2020: eAPE20200103.

TAVARES, C. Q. Dimensões do cuidado na perspectiva da espiritualidade durante a pandemia pelo novo coronavírus (COVID-19); Journal Health NPEPS. 2020 jan-jun; 5(1):1-4.

TONIN, L; et al. Recomendações em tempos de COVID-19: um olhar para o cuidado domiciliar; Rev Bras Enferm. 2020;73(Suppl 2): 1

Enviado: Novembro, 2020.

Aprovado: Novembro, 2020. 